\title{
Fundamental approach for the concept of concrete repair compatibility
}

\author{
L. Courard \\ University of Liège, Liège, Belgium
}

B. Bissonnette

Laval University, Québec, Canada

A. Garbacz

Warsaw University of Technology, Warsaw, Poland

\begin{abstract}
Before being translated in terms of physical, mechanical and chemical of materials, the initial step for evaluating compatibility is interface creation. The thermodynamic properties of the materials as well as transport mechanisms - diffusion, capillary succion - at the interface and roughness of the concrete substrate are acting from the beginning and influencing the durability of the bond strength. A review of the main parameters influencing bond development is presented on the base of the equilibrium of the surface free energies of liquid and solid, applied to the concept of repair mortars, coating and hydrophobic treatments..
\end{abstract}

\section{INTRODUCTION}

Repair works quality is depending on a lot of factors dealing with properties of the repair materials, the surface preparation of the substrate, the environment and the worker's qualification.

First of all however, it is important to verify the physico-chemical compatibility of concrete substrate and repair materials.

Surface analysis, in conjunction with surface science and applied science is a major activity contributing to the knowledge of interfacial phenomenons and, more generally, to our daily well-being through improvements in health, consumer products, environment, ... (Kinloch 1987).

But we have to define exactly what we mean through "surface analysis". In its simplest sense, we require analysis of the elemental composition of the outermost atom layer of a solid. Having defined that, the next request will be for detailed knowledge of the chemical binding state, precise sites of atoms in relation to crystal structure, surface homogeneity and the state of absorbates.

Surface free energy (Hawkins et al. 1930) is a direct measurement of intermolecular forces. The free energy in surface layer is the result of the attraction of the bulk material for the surface layer and this attraction tends to reduce the number of molecules in the surface region resulting in an increase of intermolecular distance. This increase requires work to be done and returns work to the system upon a return to a normal configuration: this explains why free energy exists and why there is a surface free energy (Zisman et al. 1950).
Before being translated in terms of physical, mechanical and chemical of materials, the initial step for evaluating compatibility is interface creation. The thermodynamic properties of the materials as well as transport mechanisms - diffusion, capillary succion - at the interface and roughness of the concrete substrate are acting from the beginning and influencing the durability of the bond strength.

\section{NEAR TO SURFACE CHARACTERISTICS}

\subsection{Principles of adhesion}

The concept of adhesion has firstly to be clearly defined because of the "duality" of the term: "on one hand, adhesion is understood as a process through which two bodies are brought together and attached - bonded - to each other, in such a way that external force or thermal motion is required to break the bond. On the other hand, we can examine the process of breaking a bond between bodies that are already in contact. In this case, as a quantitative measure of the intensity of adhesion, we can take the force or the energy necessary to separate the two bodies" (Kinloch 1987).

Adhesion has therefore two different aspects, according to whether our interest is mainly (1) in the conditions and the kinetics of contact or (2) in the separation process. The intensity of adhesion will depend not only on the energy that is used to create the contact, but also on the interaction existing in the interface zone. Generally speaking, mechanism of adhesion has to be considered from two origins: specific adhesion and mechanical interlocking (Courard 2000). 
When the materials are in contact, the effective area, that means the surface where contact really exists, will be a fundamental parameter to be taken into account to explain the adhesion process. This is the result of the wetting procedure of the solid body by the liquid phase. The wetting procedure can be explained as follows: the surface energies of the solid and the liquid interact each other and a change of the energy conditions occurs due to surface decrease of liquid/vapour and solid/vapour interfaces while a new interface (liquid/solid) is created (Dann 1970). At this point of view, contact angle is an interesting representation of this phenomenon: the lower is the contact angle, the better is the spreading on the surface and the more effective will be the intermolecular interactions at the interface (Kamusewitz et al. 1985).

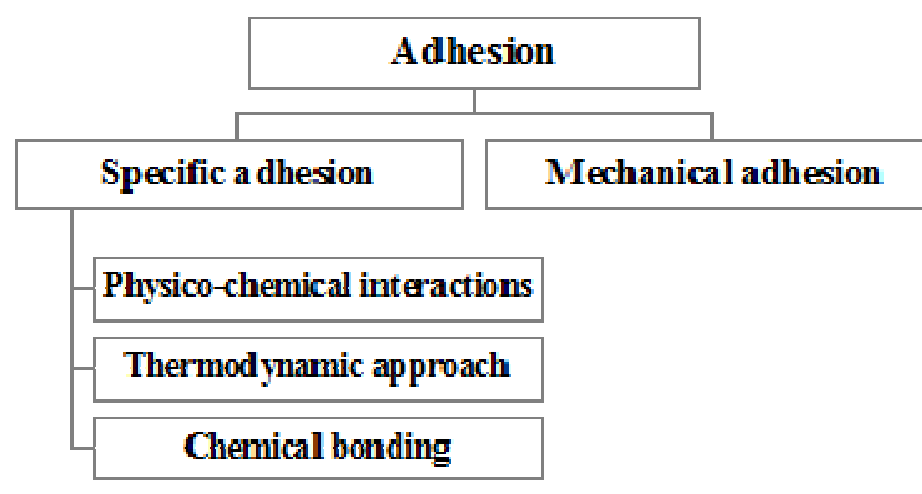

Figure 1. Principles of adhesion

The investigation concerning the behaviour of the interface between repair systems and concrete substrate have shown that quality of concrete substrate is important factor affecting adhesion in repair system and has to be evaluated prior to repair. Knowledge about synergetic effects of parameters characterizing surface quality (surface roughness, microcracking, wettability) is fundamental for concrete repair compatibility (Courard 2002).

\subsection{Microstructure of the substrate}

The physical properties are fundamental for the knowledge of the interactions at the interface. A lot of notions may be defined such as opened and accessible porosity, relative impregnation ratio or water absorption. Capillary action test seems to be very accurate to describe the behaviour of the concrete "skin" in contact with the new layer (Courard 1999) and its ability to anchor the repair material in the superficial porosity of the substrate. By means of Mercury Intrusion Porosimetry, it is possible to define the concrete skeleton and estimate the specific surface, the porous volume and the mean radiuourard 2005). But the most interesting information is the porous volume as a function of the diameter; it will be so possible to evaluate theoretically the maximum quantity of liquid each type of pore will be able to absorb (Courard et al. 2003a). Based on the law of Washburn the calculation imposes to take into account the presence of the Interfacial Transition Zone (I.T.Z.) in order to be able to correlate theory and experimental observations. The microscopical observations may be useful to determine ITZ values. These investigations will allow to calculate a coefficient of capillarity, that will take into account properties like connectivity, accessibility of the pores, tortuosity, ...

On the other hand, the nature and the concentration of the binder as well as the conditions of setting reaction are important for microscopical interpenetration: the first phenomenon observed when the new layer is laid down on the concrete substrate is the wetting of the solid surface by the liquid. The wetting is depending on two main aspects (Comyn 1992): thermodynamic (there is wetting) and kinetic (wetting has to happen as quick as possible). The kinetic aspect is physically based on the Washburn's law and is given by Equation 1.

$1_{p}^{2}=r \cdot \gamma_{L} \cdot \cos \theta \cdot t / 2 \eta$

where $l_{p}$ is the variation of penetration height, $\eta$ the viscosity of the liquid $\left(\mathrm{N} / \mathrm{m}^{2} . \mathrm{s}\right), \mathrm{r}$ the radius of the pore $(\mathrm{m}), \gamma_{\mathrm{L}}$ the superficial tension of the liquid $(\mathrm{N} / \mathrm{m}), \theta$ the contact angle and the time (s).

If one can represent concrete by a porous material with parallel capillaries of different radius, perpendicular to the liquid surface, physic's laws clearly show that the liquid will be absorbed with a higher force (equation of Laplace) for fine capillaries but at a higher speed and in higher quantity (equation of Washburn) for big capillaries. The Washburn's relation shows also that the penetration height increases when the viscosity of the liquid decreases, the contact angle decreases and the superficial tension of liquid increases.

Many remarks may be formulated about the use of Washburn's law (Courard 2000):

- $\quad$ the shape of the pore will influence the results (circular or bottle-ink for example);

- the contact angle value is not constant and depends on time and temperature;

- the pollution of the concrete surface may modify the kinetics of contact;

- the viscosity is also depending on time.

It consequently seems to be fundamental to define clearly the conditions of application in order to be able to optimise the behaviour of the liquid laid on the concrete substrate. The continuous interaction between the two bodies shows also that it is illusory to choose one without the other. These considerations indicate moreover the importance of the state of saturation of the concrete substrate before repair product application (Sasse et al. 1983).

Diffusion will also occur at the interface when contact is created. Due to its porous structure and as- 
suming it is water saturated, diffusion of ions may happen from the interstitial solution of the cement slurry or the PCC and CC mortars to the water present into the porous skeleton of the concrete and inversely. Ionic movements will participate to the creation of chemicals bonds or, at least, to chemical interactions between the substrate and the new layer; they can contribute to the development of "chemical" anchorages (Courard et al. 2003b).

\section{THERMODYNAMIC COMPATIBILITY}

\subsection{Thermodynamic equilibrium}

The superficial tension of a liquid determines its ability to wet a solid surface: an efficient spreading of the liquid on the solid increases the effective surface of contact and promotes adhesion (Courard et al. 2011). The second law of thermodynamics indicates that a system of two phases is stable if local energy is minimum.

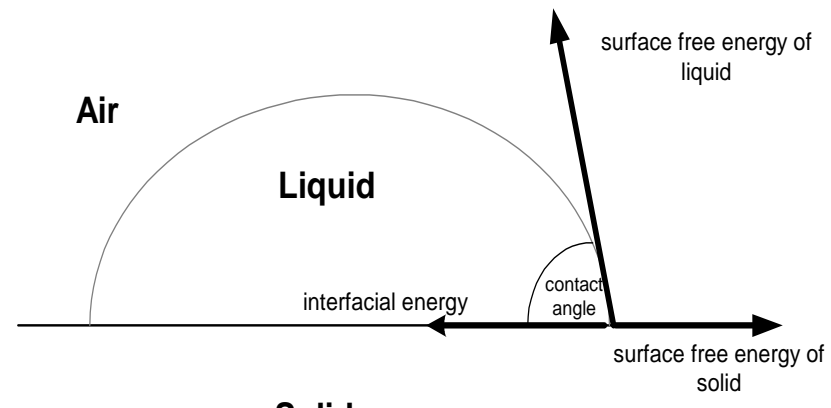

Figure 2. Wettability of a solid surface by a liquid - Young Dupré equation

For the creation and the stability of the interface between concrete and new layer, one makes the hypothesis that this minimisation of the interfacial energy corresponds to a maximum of resistance and durability of the joint (Courard 1999).

The relation between contact angle and free energies of liquid and solid is defined according to the law of Young-Dupre (Fig. 2) given by Equation 2:

$$
\gamma_{S}=\gamma_{S L}+\gamma_{L} \cdot \cos \theta
$$

where $\gamma_{S}$ is the surface free energy of solid, $\gamma_{L}$ the surface free energy of liquid and $\gamma_{S L}$ the surface free energy liquid/solid. The best spreading of liquid on the solid surface may be expressed by many criteria (Gutowski 1985) :

- minimisation of contact angle: the lower the contact angle, the better the spreading. Some additives may modify the interactions into the interstitial solution of the cement slurry, binder layer or mortar. The effects of the use of admixtures are a low decrease of viscosity and a more large decrease of contact angle values;

- free energies criteria: it is possible, from the Young-Dupre equation to conclude that, for a fixed value of $\gamma_{\mathrm{L}}$, an increase of the difference $\gamma_{S}-\gamma_{S L}$ is favourable for the wettability of the surface. This criteria is however low attractive due to the difficulty to determine $\gamma_{S}$ and $\gamma_{\mathrm{SL}}$. Another criteria is to say that $\gamma_{\mathrm{L}}$ has to be the highest as possible to obtain the lowest value of the contact angle $\theta$;

- minimization of interfacial energy or maximization of the work of adhesion. The work of adhesion represents the work necessary to separate the two bodies. These criteria are not really applicable to the situation of the creation of an interface, when liquid is spread on solid; they are more related to the resistance of the interface solid/solid, after setting or polymerization of the liquid phase.

For the analysis of the creation of the interface, it seems that the more representative criterion is the contact angle because this is a direct representation of the wettability of the surface. It is a necessary but not a sufficient condition to guarantee the efficiency of the bond.

\subsection{Relation between thermodynamic parameters and adhesion}

Some relations were found (Gutowski 1987) between the shear strength of metallic plates or glass fibres with resin and thermodynamic parameters:

$$
\tau=\mathrm{f}_{1}\left(\gamma_{\mathrm{SL} /}{\gamma_{\mathrm{L}}}^{\mathrm{C}}\right) \text { or } \tau=\mathrm{f}_{2}\left(\gamma_{\mathrm{S} /} \gamma_{\mathrm{L}}{ }^{\mathrm{C}}\right)
$$

where $\gamma_{\mathrm{L}}$ is the superficial tension of the liquid phase after hardening.

However, it is very difficult to establish such as relation between concrete and repair products due to several reasons (Possart et al. 1993):

- test devices used to measure adherence are generally not able to make distinction between the contribution of adhesion forces and loss of viscoelastic or plastic energy into concrete or the new layer;

- thermodynamic considerations on the stability of the interface solid/solid may be correlated with adherence only if rupture happens at the interface;

- the measurement of the contact angle $\theta$ with goniometer is very imprecise due to the unhomogeneity and porosity of the support. This measurement is the base for the calculation of $\gamma_{\mathrm{S}}, \gamma_{\mathrm{SL}}$ and $\mathrm{W}_{\mathrm{A}}$. More precision could be eventually obtained by working on powders.

For the analysis of the creation of the interface, it seems that the more representative criterion is the 
contact angle because it is a direct representation of the wettability of the surface. It is a necessary but not a sufficient condition to guarantee the efficiency of the bond (Possart et al. 1992).

\section{INFLUENCING PARAMETERS}

\subsection{Water}

Water is a dramatic parameter influencing the durability of repair works through the modification of surface properties of materials. The influence of temperature or relative humidity of the ambient atmosphere before the application and during the curing of the new layer are relatively well-known. It is quite common to observe:

- disturbances in the setting process;

- insufficient absorption of the binder by the substrate, due to water excess in the substrate or untimely evaporation of the solvents;

- untimely creep and/or thermal creep, in addition of setting creep,...

Coatings are designed to protect concrete against environmental aggression. Water is one of the aggressive parameters able to deteriorate the concrete structure. When water comes between the polymeric system and the concrete substrate (Courard et al. 2003), thermodynamic equilibrium is modified, in respect with particular values of surface free energies of materials. From a theoretical point of view, this means a simple generalisation of the law of Young and Dupré (Courard 2000), relatively to a new interface liquid-liquid (Equations 3 and 4). Contact angle is in this case a visible effect of the interaction between the two liquids to conquer solid surface. But what happens with spreading conditions? When equilibrium is attempted and if there is no spreading of one liquid to the detriment of the second one, equilibrium of forces means:

$\gamma_{\mathrm{SA}}=\gamma_{\mathrm{SB}}+\gamma_{\mathrm{AB}} \cdot \cos \theta$

or

$\gamma_{\mathrm{SA}}-\gamma_{\mathrm{SB}}=\gamma_{\mathrm{AB}} \cdot \cos \theta$

where $\gamma_{\mathrm{SA}}, \gamma_{\mathrm{SB}}, \gamma_{\mathrm{AB}}$ and $\theta$ are interfacial tensions between solid $\mathrm{S}$ and liquids $\mathrm{A}$ and $\mathrm{B}$, interfacial tension between liquids $\mathrm{A}$ and $\mathrm{B}$, and contact angle of these liquids on the solid surface, respectively.

It is possible to show (Kinloch 1987) that the liquid with the higher tension of adhesion $\left(\gamma_{\mathrm{x}} \cdot \cos \theta_{\mathrm{x}}\right)$ will expulse the other one from the surface. The calculation of the work of adhesion (Equation 5) can also give interesting conclusions, taking into account the variation of surface free energies in presence of water:
$\mathrm{W}_{\mathrm{x}(\mathrm{L})}=\gamma_{\mathrm{x}(\mathrm{L})} \cdot\left(1+\cos \theta_{\mathrm{x}(\mathrm{L})}\right)$

The work of adhesion is an "evaluation" of adhesion and observation of Table 1 clearly indicates the loss of adhesion when water is present at the interfaces between acrylic or epoxy resins and concrete.

Table 1. Calculation of work of adhesion for interfaces without $\left(\mathrm{W}_{\mathrm{A}}\right)$ and with $\left(\mathrm{W}_{\mathrm{AL}}\right)$ water.

\begin{tabular}{lll}
\hline Interface & $\mathrm{W}_{\mathrm{A}}\left(\mathrm{mJ} / \mathrm{m}^{2}\right)$ & $\mathrm{W}_{\mathrm{AL}}\left(\mathrm{mJ} / \mathrm{m}^{2}\right)$ \\
\hline Mortar/concrete & 87.8 & No sense \\
Acrylic/Concrete & 74.1 & 22.7 \\
Acrylic/Acrylic & 80.4 & 53.7 \\
Acrylic/Hydrophobic treatment & 52.2 & 66.7 \\
Epoxy/Concrete & 79.6 & 21.8 \\
Epoxy/Epoxy & 92.4 & 53 \\
Epoxy/Hydrophobic treatment & 56 & 42.2 \\
\hline
\end{tabular}

Thermal creep will also be produced if the application is realised in too thick layers. The degree of saturation of the substrate has also a great importance. In the example given hereafter, a PCC mortar - with dry and wet slurries - is applied on sandblasted concrete slabs characterized by different saturation levels. The adhesion strength changes in a clock-shape as a function of the water saturation level of the substrate (Table 2). The adhesion is relatively weak for low saturation levels $(<50 \%)$; it reaches classical values for saturation levels between 55 and $75 \%$. After that, we observe a decrease of the adhesion strength $(>90 \%)$.

Table 2. Adhesion of a mortar on concrete substrate vs. saturation level of concrete substrate and slurry

\begin{tabular}{lll}
\hline \multicolumn{3}{c}{ Adhesion $\left(\mathrm{N} / \mathrm{mm}^{2}\right)$} \\
\hline Saturation level & with dry slurry & with wet slurry \\
\hline 50 & 0.83 & 2.32 \\
52 & 2.80 & 2.14 \\
55 & 2.09 & 2.89 \\
70 & 2.75 & 2.65 \\
90 & 3.54 & 3.36 \\
93 & 2.13 & 3.06 \\
97 & 1.81 & 2.58 \\
100 & 1.43 & 1.48 \\
\hline
\end{tabular}

A weak saturation level produces a disturbance into the setting process of the cement while a too high saturation level acts on the attractive forces, the porosity, the kinetics of contact and, finally, the adhesion properties. One must also note that the best adhesion values are reached for a large scale of saturation levels, so that it doesn't sensitively depend on the variation of water content, except for extremal conditions. 


\subsection{Microroughness}

The surface treatment of concrete substrate is important in order to promote mechanical adhesion (Trende et al. 1998). The main problems arise from co-lateral effects of the treatment, especially due to micro-cracks parallel to the surface (Bissonnette et al. 2006). After treatment, concrete surfaces present fractal topography. As for any fractal object, it is possible to break up this surface or this profile in a sum of sub-profiles. Each sub-profile can be differentiated in terms of wavelengths; there is however no limit or precise criterion to validate the choice of decomposition method.

As mechanical interlocking is one of the basic mechanisms of the adhesion process, it is fundamental to be able to characterize the "roughness" of the substrate. Depending on local conditions of the specific building various types of surface treatments can be applied and a wild spectrum of shape and roughness can be induced (Garbacz et al. 2006).

\subsection{Human factors}

Operational factors will also induce difference in the results, depending on the tools, the competence of the worker and the manner in which he will perform the repair work. Figs. 3 and 4 show microscopical views of interface between a concrete slab and a PCC mortar applied in the same environmental conditions and from the same bag. The first worker (A) choose the techniques of pressure and smoothing while the second (B) only pressed the mortar without any finishing (12). The first view (Fig. 3) shows a lot of cracks parallel to the interface, at the interface or in the mortar, while the second is representative of a good continuity at the interface with a good spreading of air bubbles. The finishing operation (A) has induced a partial unsticking of the repair material.

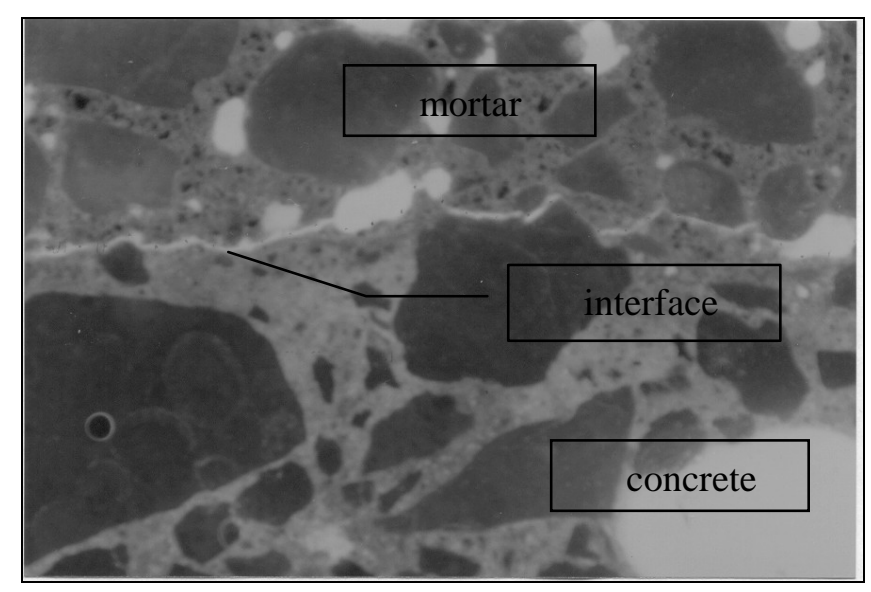

Figure 3. Microscopical view of interface between a concrete slab and a PCC mortar (operator A)

This is representative of possible destruction of the interface due to human action: forces developing ad- hesion have been destroyed by application conditions.

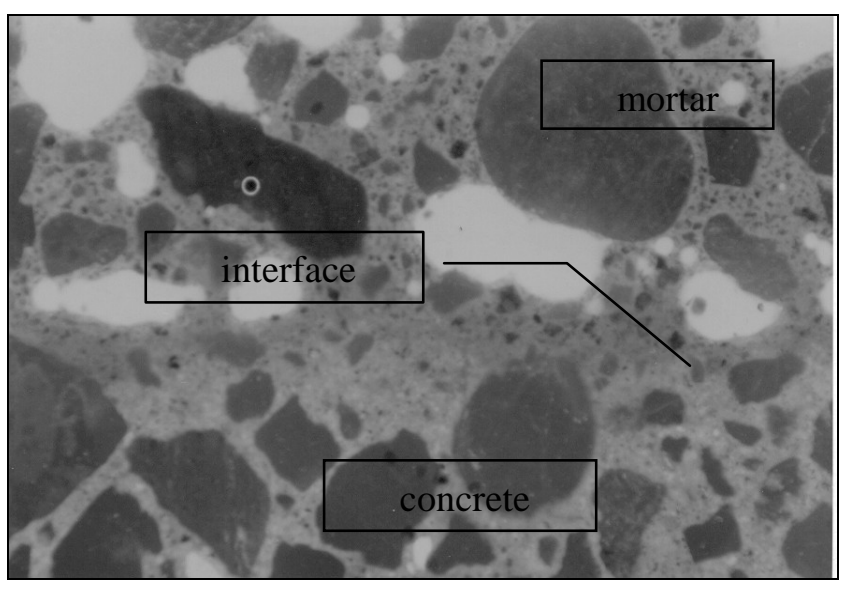

Figure 4. Microscopical view of interface between a concrete slab and a PCC mortar (operator B)

\section{CONCLUSIONS}

If there is creation of an interface, it means that there is a potential and mutual interaction between the two bodies. A physiological transposition of the situation would let us say that it is a case of partiality (from Latin word "appetentia"): this term describes the instinctive desire to all what is able to satisfy a need or something instinctive. Its application to the interface between concrete and a new layer summarizes all the parameters - physical, chemical and mechanical properties - of the substrate, the new layer and the environment that will influence the creation and the stability of this interface. Finally, that will lead to the macroscopical effect and sign of the efficiency of the cohesion: bond strength.

Compatibility between the different materials appears to be of prime importance: shrinkage, rigidity, surface roughness, viscosity, temperature, creep ...etc, will make the contact effective or not and allow interactions between the materials. But the first step remains the contact that has to be created in a very short: this is the repair speed dating!

\section{ACKNOLEDGMENTS}

This project has been financially supported by the Natural Sciences and Engineering Research Council of Canada (NSERC), the Québec FQRNT Research Fund and the industrial partners of the Industrial Chair on Durable Repair and Optimized Maintenance of Concrete Infrastructures at Laval University (BASF Building Systems, City of Montreal, City of Québec, Euclid, Hydro-Québec, King Packaged Materials, Lafarge, Ministry of Transportation of Québec, St-Lawrence Cement, W.R. Grace \& Co. Support was also provided by the Belgium (Wallonia 
Brussels International) and Quebec governments through the Wallonie-Bruxelles-Québec Scientific Cooperation Program as well as Belgium - Poland (MNiSW) Scientific Cooperation Program.

\section{REFERENCES}

Bissonnette, B., Courard, L., Vaysburd, A. and Bélair, N. (2006) Concrete removal techniques: influence on residual cracking and bond strength. Concrete International, 28(12), 49-55.

Comyn, J. (1992) Contact angles and adhesive bonding, International Journal of Adhesion and Adhesives 12(3), 145-149.

Courard, L. How to analyse thermodynamic properties of solids and liquids in relation with adhesion? in: ISAP '99, 2nd International Symposium on Adhesion between Polymers and Concrete (Eds Y. Ohama and M. Puterman, Rilem Publications), Dresden, Allemagne (1999), 9-20.

Courard, L. (2000) Parametric Study for the Creation of the Interface between Concrete and Repair Products, Materials and Structures 33, 65-72.

Courard, L. (2002) Evaluation of thermodynamic properties of concrete substrates and cement slurries modified with admixtures, Materials and Structures 35, 149-155.

(a) Courard, L. and Degeimbre, R.. (2003) A capillary suction test for a better knowledge of adhesion process in repair technology. Can. J. Civil Eng., 30(6), 1101-1110.

(b) Courard, L., Darimont, A., Degeimbre R. and Wiertz, J. (2003) Hygro-thermal application conditions and adhesion. In: ed. P. Seidler, Technische Akademie Esslingen, Fifth International Colloquium Indust-rial Floors '03, Ostfildern/Stuttgart, Germany, 37-142.

Courard, L., Michel, F. and Martin, M. (2011) The evaluation of the surface free energy of liquids and solids in concrete technology. Construction Building Materials 25(1), 260-266.

Dann J.R. (1970) Forces involved in adhesive process. I. Critical surface tensions of polymeric solids as determined by polar liquids. Journal of Colloïd Interface Science 32(2), 302331.

Fiebrich M.H., "Scientific aspects of adhesion phenomena in the interface mineral substrate-polymers", in Proceedings of 2nd Bolomey Workshop, Adherence of young and old Concrete (ed. F.H. Wittman, Aedificatio Verlag, Unterengstringen, 1994), 25-58.

Garbacz, A., Courard, L. and Kostana, K. (2006) Characterization of concrete surface roughness and its relation to adhesion in repair systems. Mater. Charact. 56, p.281-289.

Gutowski, W. (1985) Physico-chemical Criteria for Maximum Adhesion. Part I: Theoretical Concepts and Experimental Evidence. Journal of Adhesion 19, 29-49.

Gutowski, W. (1987) The relationship between the strength of an adhesivebond and the thermodynamic properties of its components. International Journal of Adhesion and adhesives 7(4), 189-98.

Harkins, W.D. and Jordan, H.F. (1930) A method for the determination of surface and interfacial tension from the maximum pull on a ring, Journal of American Chemical Society, $52,1751-72$

Kamusewitz, H. and Possart, W. (1985) The static contact angle hysteresis obtained by different experiments for the system PTFE/water, International Journal of Adhesion and Adhesives 5(4), 211-15.

Kinloch, A.J. (1987) Adhesion and Adhesives: science and technology, Chapman and Hall, London.

Possart, W. and Kamusewitz, H. (1992) Some thermodynamic considerations concerning the usual interpretation of wetting on solids, International Journal of Adhesion and Adhesives 12(1), 49-53.

Possart, W. and Kamusewitz, H. (1993) The thermodynamics and wetting of real surfaces and their relationship to adhesion, International Journal of Adhesion and Adhesives 13(2), 77-84.

Sasse, H.R. and Fiebrich, M. (1983) Bonding of polymer materials to concrete. Materials and structures 16(94), 293-301.

Tabor, D. (1981) Principles of adhesion - Bonding in cement and concrete. in Adhesion problems in the recycling of concrete, (Editor P. KREIJGER), NATO Scientific Affairs Division, 63-90.

Trende, U., and Büyüköztürk, O. 1998. Size Effect and Influence of Aggregate Roughness in Interface Fracture of Concrete Composites, ACI Materials Journal, V.95 (4), p.331338.

Zisman , W.A. and Fox, H.W. (1950). Journal of colloid science 5, 514 . 